INVESTIGACIÓN

\title{
VIGILANCIA TECNOLÓGICA E INTELIGENCIA COMPETITIVA: ESTUDIO BIBLIOMÉTRICO
}

\section{TECHNOLOGICAL SURVEILLANCE AND COMPETITIVE INTELLIGENCE: BIBLIOMETRIC STUDY}

\author{
Ph.D. Yolanda González-Castro ${ }^{\mathrm{a}}$, Msc. Msc. Omaira Manzano-Durán ${ }^{\mathrm{b}}$, Msc. Marta Milena \\ Peñaranda-Peñaranda ${ }^{c}$ \\ ${ }^{a}$ Grupo de Investigación GRINDES. Docente UNAD. Pamplona-Colombia. \\ yolanda.gonzalez@unad.edu.co \\ ${ }^{\mathrm{b}}$ Grupo de Investigación GRINDES Docente UNAD. Ocaña-Colombia. \\ omaira.manzano@unad.edu.co
}

${ }^{c}$ Universidad Francisco de Paula Santander Ocaña, Grupo de investigación GIDSE. OcañaColombia. mmpenarandap@ufpso.edu.co

Fecha de recepción: 08-09-2016

Fecha de aprobación: 02-12-2016

\begin{abstract}
Resumen: La investigación realizada aborda un paradigma cuantitativo de tipo longitudinal descriptivo con el propósito de realizar un análisis bibliométrico en el área de la vigilancia tecnológica e inteligencia competitiva que evidencia la evolución de la producción científica del tema. Se empleó la base de datos Scopus para la recolección y análisis de información y medir los diferentes indicadores de calidad, cantidad y estructura de las publicaciones, los autores y fuentes durante los años 1990 a 2014. Como resultado se encontró que la calidad y cantidad de los documentos al igual que su estructura es alta, sin embargo se requieren un mayor número de ejemplares especialmente de libros para el campo disciplinar y para la aplicación en campos específicos. De igual forma se hace necesaria una mayor producción documental en español en el tema de Inteligencia Competitiva.
\end{abstract}

Palabras clave: bibliometría, indicadores bibliométricos, inteligencia competitiva, vigilancia tecnológica.

\begin{abstract}
The research carried out deals with a quantitative longitudinal type descriptive paradigm with the purpose of performing a bibliometric analysis in the area of technological surveillance and competitive intelligence that evidences the evolution of the scientific production of the subject. The Scopus database was used to collect and analyze information and to measure the different indicators of quality, quantity and structure of publications, authors and sources
\end{abstract}


during the years 1990 to 2014. As a result it was found that the quality and quantity of The documents as well as their structure is high, nevertheless a greater number of copies are especially required of books for the disciplinary field and for the application in specific fields. Similarly increased production in Spanish documentary on the subject of competitive intelligence is necessary

Keywords: Bibliometrics, bibliometric indicators, competitive intelligence, surveillance technology.

\section{INTRODUCCIÓN}

La vigilancia tecnológica hace parte de la inteligencia competitiva en cuanto se estructura para la recolección, análisis e interpretación de la información que será difundida y aplicada a partir de procesos de inteligencia.

El presente artículo tiene como propósito presentar los resultados de un análisis bibliométrico en el campo de la vigilancia tecnológica e inteligencia competitiva, que sirva como base o estado del arte de los niveles científicos alcanzados por los investigadores del tema y emprender futuras investigaciones. La pregunta que orientó la investigación fue la siguiente: ¿Cómo ha evolucionado la investigación en vigilancia tecnológica e inteligencia competitiva en los últimos 24 años en cuanto a cantidad calidad y estructura y qué oportunidades existen en cuanto a publicaciones en este campo disciplinar? Para la búsqueda de información se contó con la base de datos de Scopus y su procesamiento y análisis a partir del paquete de Excel. Con los resultados de la información se calcularon los indicadores de cantidad (número de publicaciones), calidad (número de citaciones) y estructura (red de coautores).La metodología se centra específicamente en los indicadores de medición. Se concluye que la vigilancia tecnológica e inteligencia competitiva poseen un acervo de conocimientos en auge, alcanzando su mayor nivel de entrega literaria entre los años 2001 y 2007. Los indicadores de cantidad demuestran como los principales escritos se realizan en instituciones independientes y universidades y se presentan en su mayoría en conferencias, artículos y reseñas. Los indicadores de calidad son altos de acuerdo a los niveles de citación alcanzados, Los indicadores de estructura arrojan resultados de redes hasta de 18 personas, a través de los años la tendencia ha pasado de tres a dos autores. Los estudios se han centrado en su mayoría en el área de ingeniería sin embargo empiezan a emerger estudios con aportes a las ciencias empresariales lo que vislumbra un campo en el área gerencial específicamente en la importancia de la información para la toma de decisiones.

\section{METODOLOGÍA}

La investigación correspondió a un enfoque cuantitativo no experimental de tipo longitudinal descriptivo de los años 19902014. Las fases de la investigación fueron las siguientes: (a) Elección de la base de datos, (b) selección de términos equivalentes para la búsqueda (c) construcción de la fórmula de búsqueda de información, (d) búsqueda de información (e) análisis de indicadores de cantidad, calidad y estructura y (c) conclusiones.

El procedimiento fue el siguiente: 
1. Se eligió como base de datos Scopus para realizar la búsqueda por sus características y capacidades.

2. Se seleccionaron los términos inteligencia competitiva $\mathrm{y}$ vigilancia tecnológica en inglés y en español.

La ecuación resultante es la siguiente: "competitive intelligence" OR "surveillance technology" OR "vigilancia tecnológica" OR "inteligencia competitiva" 3. Se seleccionó el rango de 1990 a 2014 Se encontraron 5.561 registros de la búsqueda, que se analizaron en cuanto a cantidad. Para los indicadores de calidad y estructura se tomaron los 10 resultados más relevantes de los 5561 registros.

4. Se identificaron los indicadores

5. Se hizo el análisis de la información de acuerdo a las estadísticas resultantes.

\section{RESULTADOS}

\subsection{Resultados de la Revisión Teórica}

Prescott citado por Escorsa (2007) define la Inteligencia Competitiva como un proceso en el que se realiza la obtención, análisis, interpretación y difusión de información en forma sistemática. Permite dominar $\mathrm{y}$ comprender la información necesaria y hacer la toma de decisiones en el momento y por la persona específica que lo requiera (Dou, Damayanty \& Dou J.R, 2007). Su implementación repercute en la innovación, la selección de ventajas competitivas y en la misma competitividad (Rousseau, 2007).

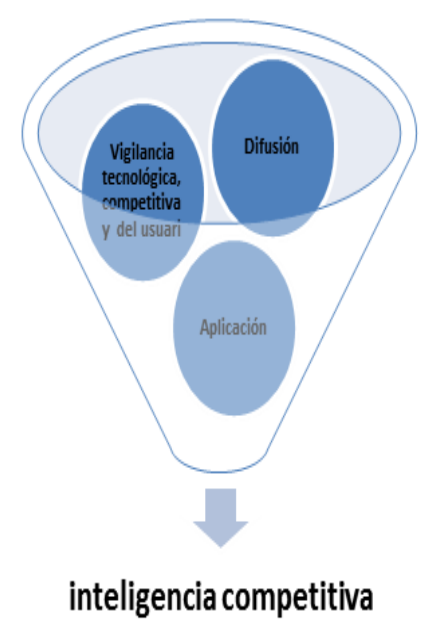

Figura 1. Inteligencia competitiva Fuente: las autoras a partir de las referencias consultadas

La vigilancia tecnológica es la primera parte de la inteligencia competitiva e incluye la fase de captura o búsqueda de la información, tratamiento y análisis de información tecnológica o científica, con el uso de métodos automáticos (Escorsa, 2007). Las fuentes de información para realizar vigilancia tecnológica generalmente se encuentra en fuentes empíricas como bases de datos e internet o fuentes tácticas como experiencias y conocimientos humanos que combinadas y organizadas en indicadores, permite gestionar la información en forma efectiva (Porter, 2007).

La Vigilancia Tecnológica y la Inteligencia Competitiva son términos modernos, para definir actividades que se realizan en forma permanente $y$ a veces en forma no consciente (Moreno, 2007). Malaver y Vargas (2007) se refieren al papel de la vigilancia tecnológica como el de identificar un camino que ha sido recorrido por los investigadores y encontrar en el camino que falta por recorrer las oportunidades y también los riesgos.

Por otra parte, Vargas y Castellanos (2005) recopilan cuatro técnicas para realizar vigilancia tecnológica y son la coocurrencia 
de palabras, los mapas tecnológicos, la minería de datos y la bibliometría. La concurrencia de palabras revela tendencias y permite comparar términos. Los mapas tecnológicos revelan las principales líneas de acción, la minería de datos extrae información y conocimientos significativos que se traducen en esquemas o modelos y la bibliometría presenta un análisis de la información encontrada y le imprime un valor agregado. Estas herramientas o técnicas se combinan a conveniencia del investigador y a la necesidad de información que se requiera.

Por otro lado, la Bibliometría es una ciencia de la ingeniería que permite el análisis cuantitativo de la producción científica a través de la literatura, estudiando la naturaleza y el curso de una disciplina científica (Camps, 2007). Es una subdisciplina de la cienciometría y suministra información sobre los resultados del proceso investigador, su volumen, evolución, visibilidad y estructura. Permitiendo valorar la actividad científica, y el impacto tanto de la investigación como de las fuentes (Escorcia, 2008).

Durieux y Gevenois citado por Cadavid, Awad y Franco (2012) afirman que la técnica del análisis bibliométrico recoge un conjunto de métodos matemáticos y estadísticos que se emplean para analizar y medir las publicaciones científicas permitiendo una adecuada toma de decisiones.

De acuerdo con Vallejo y Ruiz (2005) los indicadores bibliométricos se pueden dividir en dos grandes grupos: a) los indicadores cuantitativos de la actividad científica que incluye la cantidad de publicaciones y permite visualizar el estado real de la ciencia, y b) los indicadores de impacto que se basan en la cantidad de citas que se obtienen de los trabajos, y caracterizan la importancia del documento de acuerdo al reconocimiento otorgado por otros investigadores.

Por otro lado Durieux y Gevenois (2010) argumentan que existen tres tipos de indicadores bibliométricos: a) de cantidad que miden la productividad de un investigador b) de calidad por estar orientados a medir el número de citaciones y c) de estructura, que miden la conectividad entre las publicaciones, los autores y las áreas de conocimiento.

El análisis bibliométrico se encuentra limitado por la información disponible (Rueda, Gerdsri \& Kocaoglu, 2007). Por lo tanto es de gran relevancia evaluar cuál base de datos usar para medir la producción académica. Es así como los criterios de cobertura, de desempeño, accesibilidad y flexibilidad de los operadores de búsqueda favorecen a la base de datos Scopus e ISI por encima de las bases de datos disponibles (Hall, 2011).

\subsection{Resultados del análisis bibliométrico}

En cuanto al indicador de cantidad "número de publicaciones por año", se obtuvo la información que se aprecia en la figura 2.

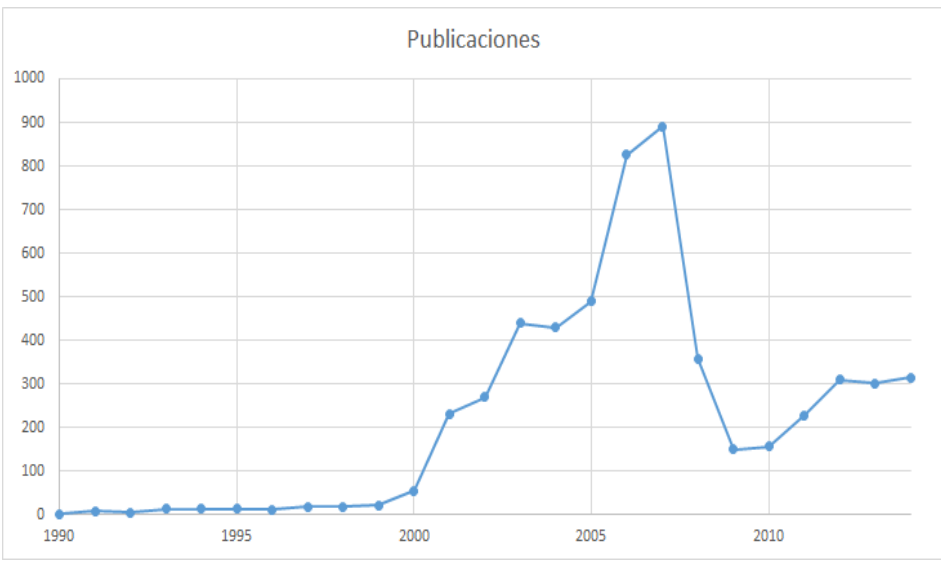

Figura 2. Publicaciones de vigilancia tecnológica o inteligencia competitiva de 1990 al 2014

Fuente: Las autoras a partir de la base de datos Scopus 
Tras largos años de introducción lenta, del 2001 al 2007 hubo un alto auge de trabajos relacionados con vigilancia tecnológica e inteligencia competitiva. Con una disminución a partir de 2008.

Por otro lado, como se observa en la figura 3, la fuente de mayor publicación en temas de vigilancia tecnológica e inteligencia competitiva a lo largo de los años es Procedings of SPIE the International society for optical engineering. A partir del 1996 al 2008 existen publicaciones en la International Journal of Thechnology Management. Del 2001 al 2007 existen amplias publicaciones en IEEE International Engineering Management Conference. Entre el 2003 y el 2005 se presentaron publicaciones de Chemical Market Reporter y en los últimos años aparecen publicaciones en el Journal of Intelligence Studies in Business.

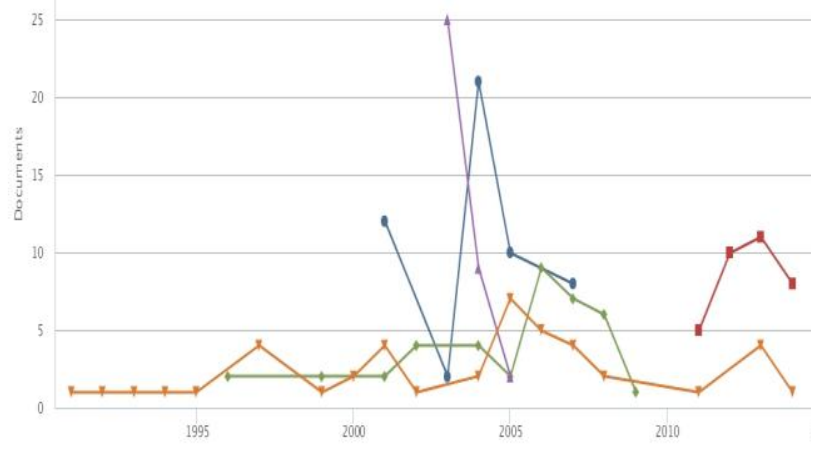

— IEEE International Engineering Management Conference $*$ Journal of Intelligence Studies in Business + International Joumal of Technology Management + Chemical Market Reporter + Proceedings of SPlE the International Society for Optical Engineering

Figura 3. Fuentes de mayor publicación de vigilancia tecnológica o inteligencia competitiva

Fuente: Las autoras a partir de la base de datos Scopus

Asimismo, se pueden apreciar en la figura 4 los diez investigadores que más publicaciones tienen sobre el tema, siendo el primero de ellos Yeoh William con doce publicaciones. Al respecto, estos diez investigadores publican sólo el $11 \%$ de la producción académica sobre vigilancia tecnológica e inteligencia competitiva.

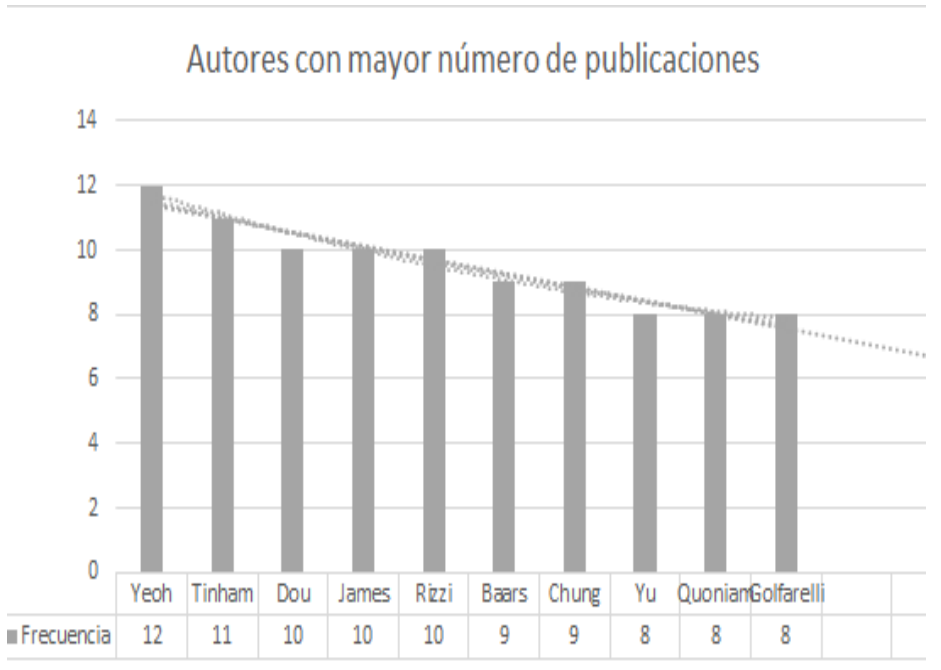

Figura 4. Autores con mayor número de publicaciones

Fuente: Las autoras a partir de la base de datos Scopus

Tabla 1.

$\mathrm{H}$-index, citaciones y coautores

\begin{tabular}{|l|c|c|c|c|}
\hline $\begin{array}{c}\text { Autores/ } \\
\text { indicadores }\end{array}$ & $\begin{array}{c}\text { Docu- } \\
\text { mentos }\end{array}$ & $\begin{array}{c}\text { h- } \\
\text { index }\end{array}$ & $\begin{array}{c}\text { Citacio } \\
\text { nes }\end{array}$ & $\begin{array}{c}\text { Co- } \\
\text { autores }\end{array}$ \\
\hline $\begin{array}{l}\text { 1.Yeoh, } \\
\text { William }\end{array}$ & 66 & 6 & 181 & 74 \\
\hline $\begin{array}{l}\text { 2.Tinham, } \\
\text { Brian J. }\end{array}$ & 653 & 3 & 35 & 6 \\
\hline $\begin{array}{l}\text { 3.James, Dick } \\
\text { G }\end{array}$ & 28 & 4 & 52 & 13 \\
\hline $\begin{array}{l}\text { 4.Rizzi, } \\
\text { Stefano }\end{array}$ & 82 & 14 & 1008 & 73 \\
\hline 5.Dou, Henri & 38 & 6 & 172 & 33 \\
\hline 6.Baars, & 25 & 4 & 104 & 18 \\
\hline
\end{tabular}




\begin{tabular}{|l|c|c|c|c|}
\hline Henning & & & & \\
\hline $\begin{array}{l}\text { 7.Chung, } \\
\text { Wingyan }\end{array}$ & 46 & 12 & 619 & 50 \\
\hline $\begin{array}{l}\text { 8.Rothberg, } \\
\text { Helen N. }\end{array}$ & 22 & 2 & 28 & 4 \\
\hline $\begin{array}{l}\text { 9.Golfarelli, } \\
\text { Matteo }\end{array}$ & 67 & 14 & 857 & 58 \\
\hline $\begin{array}{l}\text { 10. Wright, } \\
\text { Sheila }\end{array}$ & 11 & 6 & 91 & 8 \\
\hline
\end{tabular}

Fuente: Las autoras a partir de la base de datos Scopus

Al analizar la relación entre la producción literaria y su aporte al campo de conocimiento (ver Tabla 1) es de resaltar la calidad de los aportes de Rizzi Stefano, por los impactos que ha logrado al alcanzar un alto número de citaciones con pocos escritos. Igual ocurre con Matteo, Golfarelli y Wingyan, Chung, aunque en menor medida (ver figura 5), donde los números del eje x están alineados con la Tabla 1.

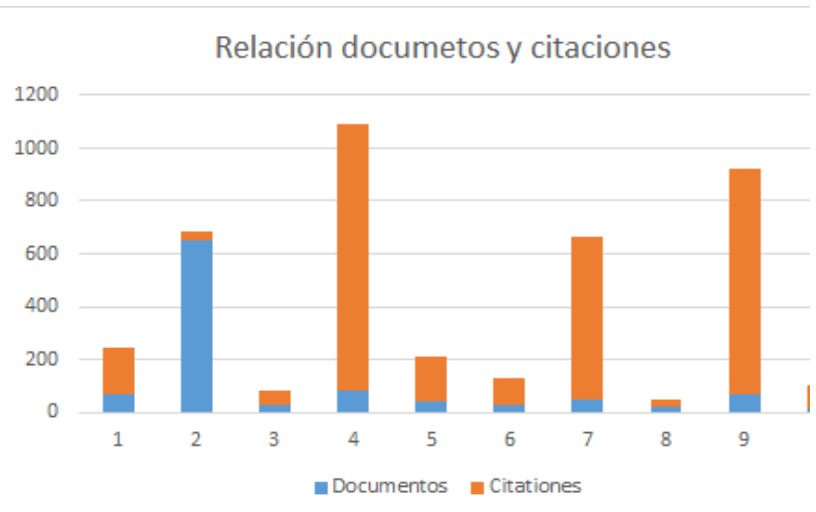

Figura 5. Relación documentos y citaciones

Fuente: Las autoras a partir de la base de datos Scopus

Los resultados del $h$-index (ver Tabla 1), muestran el avance e impactos de los investigadores en el área disciplinar y permite identificar a Rizzi, Stefano; Golfarelli, Matteo y Chung, Wingyan como los de más alta citación.
En referencia a la afiliación, comunidades autónomas y universidades son las que aportan al conocimiento en vigilancia tecnológica e inteligencia competitiva. Las más representativas son: International Business Machines, IEEE, Harbin Institute of Technology y Tsinghua University (ver figura 6).

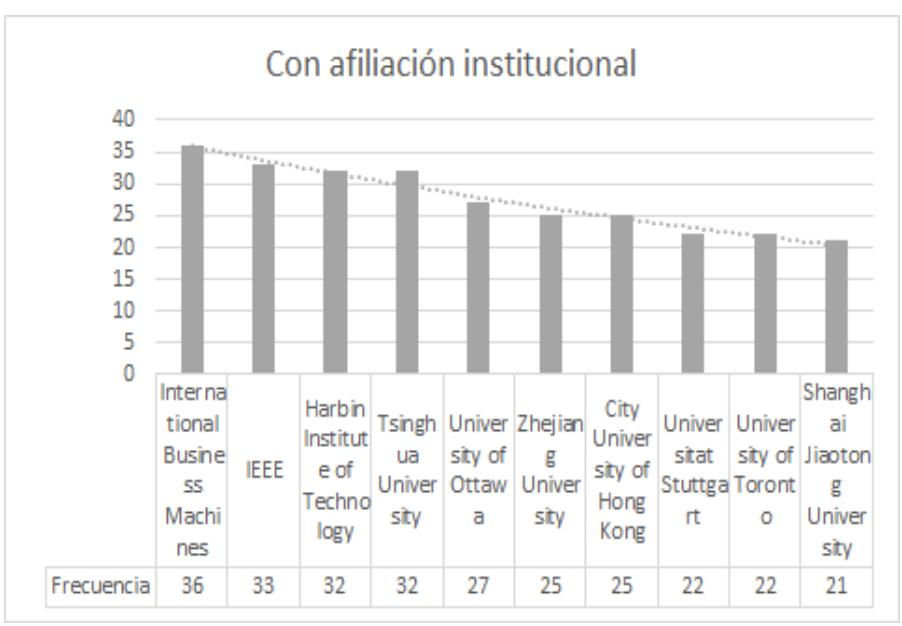

Figura 6. Afiliación institucional

Fuente: Las autoras a partir de la Base de datos Scopus

Por otro lado, los países donde más se publican trabajos de vigilancia tecnológica e inteligencia competitiva son en Estados Unidos seguido por China y el Reino Unido.

Del mismo modo, se han realizado conferencias y espacios para la presentación de aportes en el tema de vigilancia tecnológica e inteligencia competitiva. Las principales producciones literarias son documentos de conferencias, artículos y 
reseñas. Existen pocos libros en relación al tema.

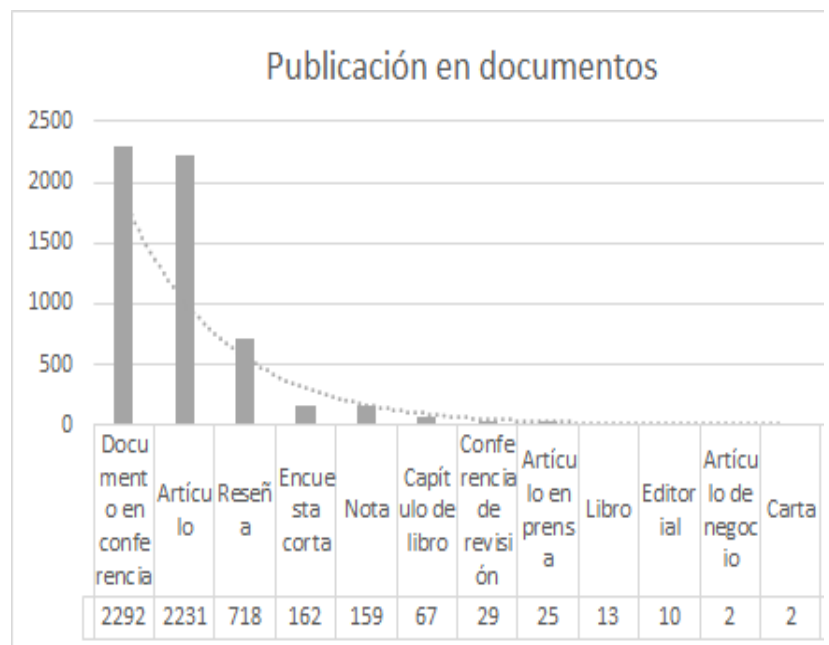

Figura 7. Publicaciones en documentos Fuente: Base de datos Scopus

Además, se pudo identificar que el tema de vigilancia tecnológica e inteligencia competitiva tiene una amplia literatura especialmente en ingeniería, ciencias de la computación y administración de negocios.

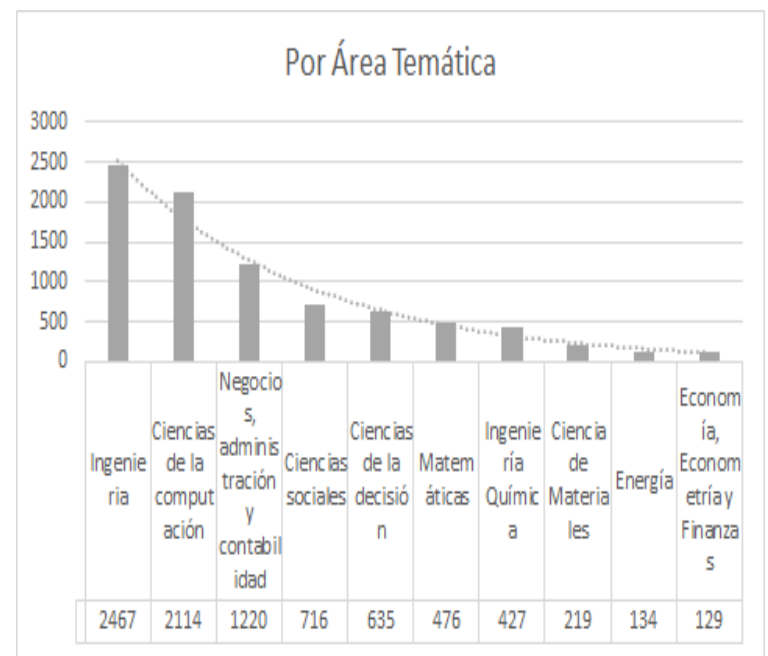

Figura 8. Área temática de la vigilancia tecnológica y la inteligencia competitiva

Fuente: Base de datos Scopus

Finalmente, al revisar las redes de autores en torno a la producción se puede apreciar que los autores con mayor cantidad de publicaciones tienden a publicar en pequeñas redes (parejas o tríos) mientras que las redes con gran cantidad de autores tienen pocas publicaciones (ver figura 9)

Tabla 2.

Redes en torno a la producción

\begin{tabular}{|c|c|c|c|}
\hline Coautores & Publicaciones & Coautores & Publicaciones \\
\hline $\mathbf{1}$ & 499 & $\mathbf{1 0}$ & 2 \\
\hline $\mathbf{2}$ & 582 & $\mathbf{1 1}$ & 9 \\
\hline $\mathbf{3}$ & 494 & $\mathbf{1 2}$ & 0 \\
\hline $\mathbf{4}$ & 239 & $\mathbf{1 3}$ & 0 \\
\hline $\mathbf{5}$ & 94 & $\mathbf{1 4}$ & 2 \\
\hline $\mathbf{6}$ & 39 & $\mathbf{1 5}$ & 0 \\
\hline $\mathbf{7}$ & 23 & $\mathbf{1 6}$ & 0 \\
\hline $\mathbf{8}$ & 8 & $\mathbf{1 7}$ & 0 \\
\hline $\mathbf{9}$ & 7 & $\mathbf{1 8}$ & 2 \\
\hline
\end{tabular}

Fuente: Las autoras a partir de la base de datos Scopus 


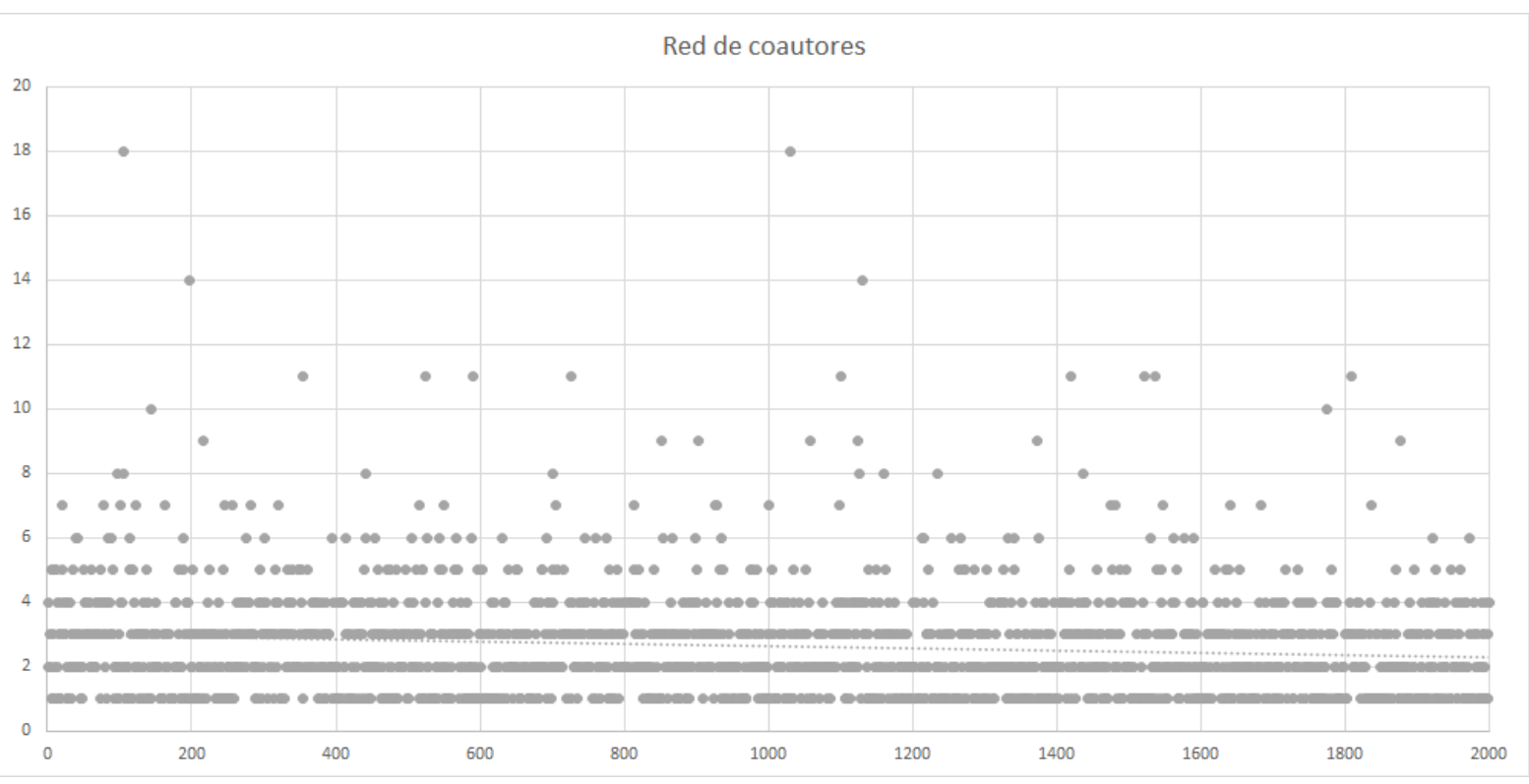

Figura 9. Redes en torno a la producción Fuente: Las autoras a partir de la base de datos Scopus

\section{CONCLUSIONES}

En esta investigación se realizó un análisis bibliométrico con el empleo de la base de datos Scopus que permitió determinar tendencias de investigación en el área de la vigilancia tecnológica y la inteligencia competitiva. La bibliometría se ha convertido en una herramienta que permite calificar y medir el proceso de generación y transferencia del conocimiento así como el impacto generado en la comunidad científica.

El área de la vigilancia tecnológica y la inteligencia competitiva tuvo un gran crecimiento a partir del 2001 hasta el 2007. La producción se encuentra centralizada en países como Estados Unidos, China y Reino Unido y en el idioma inglés.

El tema sigue siendo de mucha relevancia y continua en permanente investigación pues mayor parte de la socialización del conocimiento se realiza en conferencias, artículos y reseñas.

Los autores con mayor cantidad de publicaciones como Tinham, Brian J. no son los autores más citados, evidenciando que existe una baja correlación entre cantidad y la calidad de las investigaciones en el tema. Sin embargo con otros autores como Rizzi Stefano la situación es contraria pues ha logrado alcanzar un alto número de citaciones con pocos escritos. Las redes de autores de estas publicaciones tienen a ser pequeñas y a pasar de tres a dos autores.

El análisis realizado dejó por fuera patentes así como también algunas consideraciones sobre minería de datos. Un análisis que integre estos componentes podría dar una orientación sobre campos emergentes para futuras investigaciones.

\section{BIBLIOGRAFÍA}

Cadavid, L., Awad, G. y Franco, C. (2012). Análisis bibliométrico del campo modelado de difusión de innovaciones. Estudios gerenciales, 28, 213-236 
Camps D. (2007). Estudio bibliométrico general de colaboración y consumo de la información en artículos originales de la revista Universitas Médica, período 2002 a 2006, Universitas Médica; 48(4): 358-365

Dou, H., Damayanty, M. and Dou J.R, H. (2007). Inteligencia competitiva, alianzas público-privadas, innovación, política de clusters y desarrollo regional. En: La inteligencia competitiva factor clave para la toma de decisiones estratégicas en las organizaciones, 1st ed. Madrid: Fundación Madrid para el Conocimiento, pp.24-39.

Escorcia, T. (2008). El análisis bibliométrico como herramienta para el seguimiento de publicaciones científicas, tesis y trabajos de grado. Recuperado de: http://www.javeriana.edu.co/biblos/tesi s/ciencias/tesis209.pdf

Escorsa, P. (2007). Introducción. En: La inteligencia competitiva factor clave para la toma de decisiones estratégicas en las organizaciones, 1st ed. [online] Madrid: Fundación Madrid para el Conocimiento, pp.10-22.

Hall, M. (2011). Publish and perish? Bibliometric analysis, journal ranking and the assessment of research quality in tourism. Tourism Management, 32(1), 16-27

INTEC(2007). La inteligencia competitiva factor clave para la toma de decisiones estratégicas en las organizaciones. Recuperado de: http://www.madrimasd.org/informacio nidi/biblioteca/publicacion/doc/35_IN TEC.pdf

Malaver, F \& Vargas, M. (2007). Vigilancia tecnológica y competitividad sectorial. 1st ed. Bogotá: Favegraf, 330 páginas.

Moreno, L. (2007). La información como herramienta de la Vigilancia Estratégica. Revista Iberoamericana sobre usuarios de información, 35(1), $1-11$.

Porter, A.. (2007). I La Innovación Abierta 1 (Open Innovation) mediante la aplicación de la minería tecnológica 2 (Tech Mining). En: La inteligencia competitiva factor clave para la toma de decisiones estratégicas en las organizaciones, 1st ed. Madrid: Fundación Madrid para el Conocimiento, pp.53-74.

Rousseau, j. (2007). Inteligencia territorial y "Movinnova". En: La inteligencia competitiva factor clave para la toma de decisiones estratégicas en las organizaciones, 1st ed. Madrid: Fundación Madrid para el Conocimiento, pp.94-105.

Rueda, G., Gerdsri, P. \& Kocaoglu, D.F. (2007). Bibliometrics and Social Network Analysis of the Nanotechnology Field. Management of Engineering and Technology, Portland International Center for, 2905-2911.

Vargas, \& Castellanos, O. (2005). Vigilancia como herramienta de innovación y desarrollo tecnológico. Caso de aplicación: sector de empaques plásticos flexibles. Revista Ingeniería e Investigación. 25(2), 32-41.

Vallejo-Ruiz M.(2005). Estudio Longitudinal De La Producción Española De Tesis Doctorales En educación Matemática (1975-2002), Tesis Doctoral. Departamento de Métodos de Investigación y Diagnóstico en Educación. Universidad de Granada, Granada, España, 2005, 535p.p. 